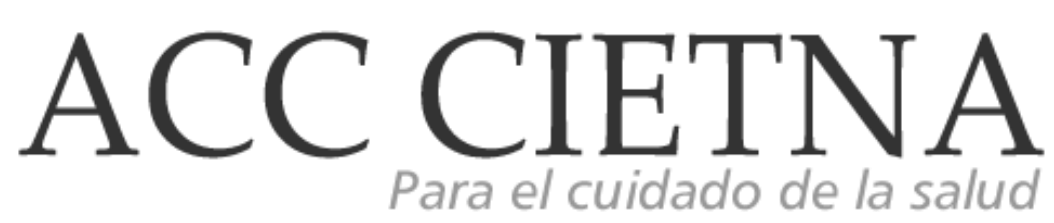

https://doi.org/10.35383/cietna.v3i1.53

\title{
Conocimientos y prácticas en la prevención de riesgos biológicos de las enfermeras del hospital Belén Lambayeque
}

\author{
Alarcón Bautista María Doris ${ }^{1}$, Rubiños Dávila Shirley Cinthiaㄹ, Guzmán Tello Socorro Martina ${ }^{3}$
}

\begin{tabular}{ll}
\hline INFORMACIÓN DEL ARTíCULO & RESUMEN \\
\hline Historia del artículo: & La investigación cuantitativa, de diseño correlacional y de corte \\
Aceptado el 18 de abril de 2016 & transversal, se realizó en el Hospital provincial docente Belén de \\
& Lambayeque, tuvo como objetivo principal, determinar la relación que \\
\hline Palabras claves: & existe entre conocimientos y prácticas en la prevención de riesgos \\
Conocimientos & biológicos en las enfermeras del Hospital Belén. El estudio estuvo \\
Prácticas & constituido por una población muestral de 43 enfermeras que laboran \\
Prevención & en diferentes servicios de la institución, se empleó la técnica de campo; \\
Enfermería & el cuestionario y la lista de cotejo, como instrumentos de recolección \\
& de datos. Los resultados fueron analizados según la prueba de chi- \\
& cuadrado, donde se concluyó que: No existe relación significativa entre \\
& conocimientos y prácticas en la prevención de riesgos biológicos de las \\
& enfermeras del Hospital Belén-Lambayeque. Con respecto al \\
& conocimiento de riesgos biológicos: Del 100\% (43) de enfermeras que \\
& laboran en dicho nosocomio, $67.44 \%$ tienen un nivel de conocimiento \\
& regular y solo el $6.98 \%$ poseen un buen nivel de conocimiento en la \\
& prevención de riesgos biológicos. Según las prácticas en riesgos \\
& biológicos: El $4.65 \%$ de las enfermeras, tienen buena práctica y el \\
& $39.53 \%$ tienen una práctica deficiente en la prevención de riesgos \\
biológicos.
\end{tabular}

\footnotetext{
${ }^{1}$ Licenciada en Enfermería. Enfermera con trabajo independiente, Chiclayo, Perú. Email: maria_doris15@hotmail.com

${ }^{2}$ Licenciada en Enfermería. Enfermera con trabajo independiente, Chiclayo, Perú.

${ }^{3}$ Magister en Ciencias de Enfermería. Docente en la Escuela de Enfermería de la Universidad Católica Santo Toribio de Mogrovejo, Chiclayo, Perú. Email: sguzman@usat.edu.pe
} 


\section{Knowledge and practices in the prevention of biological hazards of hospital nurses Belen Lambayeque}

\section{ABSTRACT}

Keywords:

Knowledge

Practices

Prevention

Biohazard

Nursing
Quantitative research, correlational design and cross-section was performed at the Hospital provincial teaching Bethlehem Lambayeque main objective was to determine the relationship between knowledge and practice in the prevention of biological hazards in nurses Hospital Belen. The study consisted of a sample population of 43 nurses working in different departments of the institution, the field technique was used; the questionnaire and checklist, as data collection instruments. The results were analyzed by the chi-square, where it was concluded that: There is no significant relationship between knowledge and practice in the prevention of biological hazards of nurses Belén-Lambayeque Hospital. With regard to knowledge of biohazards: From 100\% (43) of nurses who work in that hospital, $67.44 \%$ have a regular knowledge level and only $6.98 \%$ have a good level of knowledge in the prevention of biological hazards. According biohazards practices: The $4.65 \%$ of nurses have good practice and $39.53 \%$ had poor practice in the prevention of biological hazards.

\section{Introducción}

A pesar de ser imprescindibles las condiciones de seguridad para la realización de cualquier actividad laboral, es común encontrarnos con situaciones peligrosas, en las cuales las exigencias legales para el control de los riesgos ocupacionales no se tienen en cuenta por negligencia, causando así accidentes y enfermedades en los trabajadores. Dentro de las áreas de actuación laboral está el hospital, donde se realizan actividades insalubres, es decir, aquellas que por su naturaleza, condiciones o métodos de trabajo exponen al trabajador a agentes nocivos para su salud.1,2

Según la Organización Internacional del Trabajo (OIT), en el 2011, informa que alrededor de 337 millones de personas son víctimas de accidentes y enfermedades laborales cada año. ${ }^{3}$ Así mismo la organización mundial de la salud (OMS) indica que los accidentes más frecuentes ocurren con el personal de salud, un tercio de todas las lesiones se presentan en el personal de enfermería debido al uso inadecuado de las medidas de bioseguridad, además estima que la carga global de enfermedades por exposición ocupacional entre el personal de salud corresponde en un $40 \%$ a las infecciones por hepatitis B y C y un $2.5 \%$ de las infecciones por $\mathrm{VIH} .{ }^{4}$

El Perú no es ajeno a esta realidad, la investigación "Accidentes con fluidos biológicos en el Hospital Nacional Dos de Mayo", 2006, evidencia que las enfermeras ocupan el segundo lugar dentro del grupo ocupacional de los accidentes de este tipo, específicamente el $81 \%$ con material punzo cortante: agujas hipodérmicas, y un $17 \%$ por salpicaduras en ojos y en mucosas; además indica que los servicios donde ocurrieron los accidentes laborales fueron: emergencia 33\%, sala de operaciones central 10\%; y las circunstancias donde ocurrieron estos accidentes durante el procedimiento quirúrgico $37 \%$, siendo esta realidad un problema que atenta contra el trabajo digno de los profesionales de la salud. ${ }^{5}$ 
El profesional de enfermería en su quehacer diario se encuentra expuesto a múltiples riesgos en el medio sanitario, siendo los biológicos los más frecuente, en las que existe la posibilidad de entrar en contacto con sangre o fluidos corporales y sobre todo, de sufrir inoculaciones accidentales al manipular objetos cortantes y punzantes, o exposiciones de piel y mucosas. 6,7 De ahí que, las infecciones adquiridas tras la exposición accidental del profesional sanitario entre ellos los enfermeros con fluidos biológicos, constituyen un grupo de enfermedades profesionales con importantes implicaciones sociales, laborales, legales y económicas, por ello la prevención en transmisión de agentes infecciosos en una institución de salud, es esencial para una gestión activa de la seguridad y la salud en el trabajo, todo esto se respalda en el cumplimiento de las normas de bioseguridad específicas para cada área, en las actividades de vigilancia diseñadas para el control de infecciones $y$ en los programas de salud $y$ seguridad para los trabajadores, los cuales son más efectivos si se cuenta con una estrategia permanente de refuerzo del conocimiento en los profesionales. ${ }^{1,6}$

Al respecto, durante las prácticas hospitalarias las investigadoras observaron que las enfermeras de los diferentes servicios del hospital, estuvieron en mayor exposición al manejo de fluidos biológicos y/o riesgo a enfermedades ocupacionales debido a los procedimientos que realizaban: canalización de vías endovenosas, aspiración de secreciones, transfusiones sanguíneas, curación de heridas, etc.

No sólo al mayor contacto con sangre, secreciones en general, agujas, jeringas e instrumental contaminado; si no también a las condiciones laborales, es decir la presencia de material y equipos que permitan condiciones óptimas para proteger al profesional de enfermería del riesgo a sufrir accidentes laborales por inadecuadas prácticas en la prevención de riesgos biológicos.
En tal sentido, en las instituciones del ministerio de salud (MINSA), se percibe una escasa implementación con respecto a los quipos de bioseguridad como: mandiles, mascarillas, guantes, lentes protectores, etc. Además existe una gran demanda de pacientes en estas instituciones que no cuentan con recursos económicos, asumiendo ellos mismos la obtención del material para el paciente y la protección de las enfermeras, algunos no adquieren todos los materiales, por ende los profesionales de enfermería no tienen una adecuada protección al realizar los procedimientos por no contar con equipos de bioseguridad predisponiéndoles en muchas ocasiones a adquirir enfermedades por contacto con fluidos corporales derivados de la atención del paciente, dificultando la calidad de los cuidados de enfermería, como también su salud. Sin embargo esto sería minimizado si en estas instituciones hubiera una adecuada distribución de material así como también realizando capacitaciones cada ciertos periodos de tiempo comprometiendo la asistencia de los trabajadores, así mismo proporcionando manuales que estén a la disposición de todos los trabajadores protegiendo de esta manera su salud e integridad.

A esto se le suma una deficiente responsabilidad y prácticas por parte de las enfermeras en la prevención de agentes biológicos en dicho nosocomio. Al respecto algunas enfermeras manifestaron: "hay muchos pacientes, no hay material, no es de riesgo", realizan sus actividades con cuidado, el paciente no está infectado, etc. Las enfermeras como parte importante en la atención de pacientes son las encargadas de proporcionarles minuciosamente los cuidados de enfermería a todos los que le son asignados, para ello requieren conocimientos, habilidades $y$ actitudes que le permiten desarrollar una actividad digna y abnegada tomando en cuenta sus principios éticos y morales.

Este estudio se realizó por iniciativa e interés de las investigadoras debido al aumento significativo 
de enfermedades infectocontagiosas, que de manera directa e indirecta afecta a los enfermeros que brindan cuidados, por déficit de conocimiento o inadecuadas prácticas en la prevención de riesgos biológicos, y en el tiempo convertirse en un eslabón más de la cadena de transmisión, siendo la prevención, la única medida posible contra estas enfermedades. Para ello, se direccionaron los siguientes objetivos específicos: Determinar el nivel de conocimientos de las enfermeras en la prevención de riesgos biológicos y determinar el nivel de prácticas de las enfermeras en la prevención de riesgos biológicos en el Hospital Belén.

En este sentido, la investigación buscó promover una conducta reflexiva en la prevención de riesgos biológicos, aplicando los conocimientos en el cuidado brindado a la persona, familia $y$ comunidad, teniendo en cuenta que la enfermera cumple un rol muy importante en el control de las infecciones intrahospitalarias.

Además, el estudio fue relevante a nivel institucional, proponiendo la participación activa de todas las enfermeras con cambios de actitudes mediante la capacitación (programa educativo) y supervisión de las prácticas correctivas en la prevención de riesgos biológicos, el cual debe estar acorde con el avance científico y tecnológico, involucrando objetivos y normas definidas logrando un ambiente de trabajo ordenado, seguro que conduzca simultáneamente a mejorar la calidad de atención, reducir los incrementos de costos alcanzando los óptimos niveles de funcionalidad confiable y así favorecer la.

\section{Metodología}

El estudio, fue una investigación cuantitativa, de diseño correlacional y corte transversal.8,9 Se realizó en Hospital Provincial Docente Belén, que se encuentra ubicado en departamento Lambayeque, es un hospital de nivel III, brinda servicios integrales accesibles de salud individual y colectiva, en el proceso de salud - enfermedad de la población.

La población estuvo constituida por 43 enfermeras de los diferentes servicios, entre ellos: medicina, ginecología, traumatología, pediatría, neonatología, centro quirúrgico, cirugía y emergencia, por lo que no se empleó fórmula para obtener una muestra de estudio, por ser una población muestral. 8

Para la recolección de datos se utilizaron el cuestionario y la lista de cotejo. El cuestionario estuvo estructurado en cuatro partes: La primera, estuvo relacionada con las características generales de las enfermeras; la segunda, con los riesgos biológicos, con 6 preguntas; la tercera, a medidas de bioseguridad y consta de 11 preguntas; por último, el manejo de residuos hospitalarios, con 3 preguntas. De esta forma la lista de cotejo fue elaborado por las autoras, relacionándolo con el contenido del cuestionario, se empleó para cotejar cada una de las acciones realizadas por las enfermeras.

La muestra se determinó, teniendo en cuenta criterios de inclusión tales como: Enfermeras que laboran en los diferentes servicios del hospital y enfermeras que estén laborando como mínimo 6 meses en los servicios. De exclusión: Enfermeras que estén laborando en el área de administración y enfermeras que no desean participar en la investigación

Para la confiabilidad del cuestionario se utilizó la prueba de coeficiente alfa de Cronbach obteniendo como resultado 0.87 , lo cual indicó una buena confiabilidad del instrumento. Al mismo tiempo la lista de cotejo se analizó con la prueba Kuder y Richardson o coeficiente KR-20, que evalúa la consistencia interna del instrumento, siendo aplicable solo a ítems dicotómicos, que pueden ser calificados con 0 y 1.10 Obteniendo como resultado 0.69 lo que indica una buena confiabilidad. 
Inicialmente se explicó a los participantes del estudio en qué consistió la investigación, previo a la firma del consentimiento informado. Al finalizar la recolección de datos, se procedió a crear la base de datos en el programa de Excel, mediante un proceso de análisis de cada variable, así mismo se construyeron tablas de frecuencia univariadas $y$ bivariadas, así como gráficos estadísticos de acuerdo a los objetivos planteados en la investigación.

Para determinar la existencia o no de relación entre las variables se aplicó la prueba Chi cuadrado, la cual dio como resultado la aceptación de la hipótesis nula: "No existe relación significativa entre conocimientos y las prácticas en la prevención de riesgos biológicos de las enfermeras del hospital Belén - Lambayeque.

\section{Resultados}

A continuación se presentan los resultados de la investigación de acuerdo a los objetivos formulados en el proyecto.

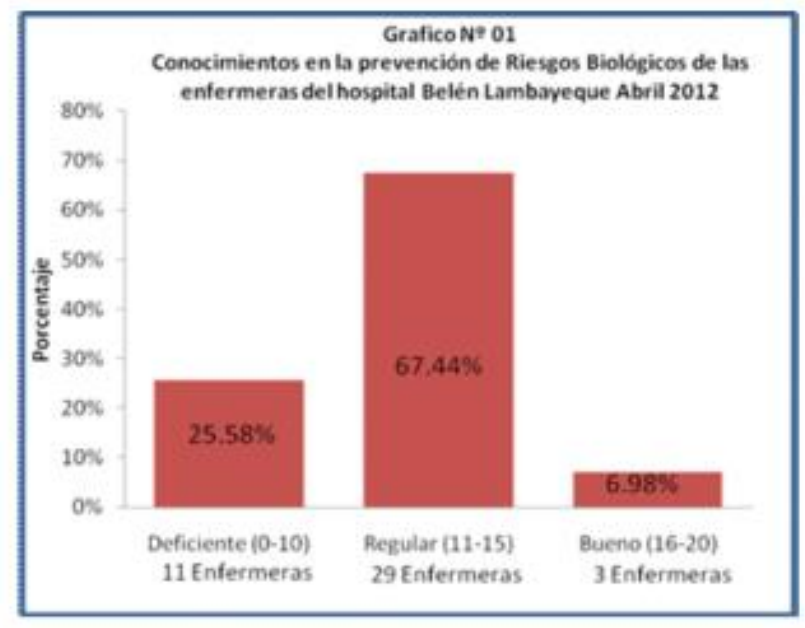

Fuente: Cuestionario realizados a las enfermeras del hospital Provincial Docente Belén Abril 2012

Según el gráfico $\mathrm{N}^{\circ} 01$ del 100\% de enfermeras que laboran en el hospital Belén-Lambayeque, el $67.44 \%$ tiene un conocimiento regular y solo el $6.98 \%$ tienen conocimiento bueno en la prevención de riesgos biológicos.

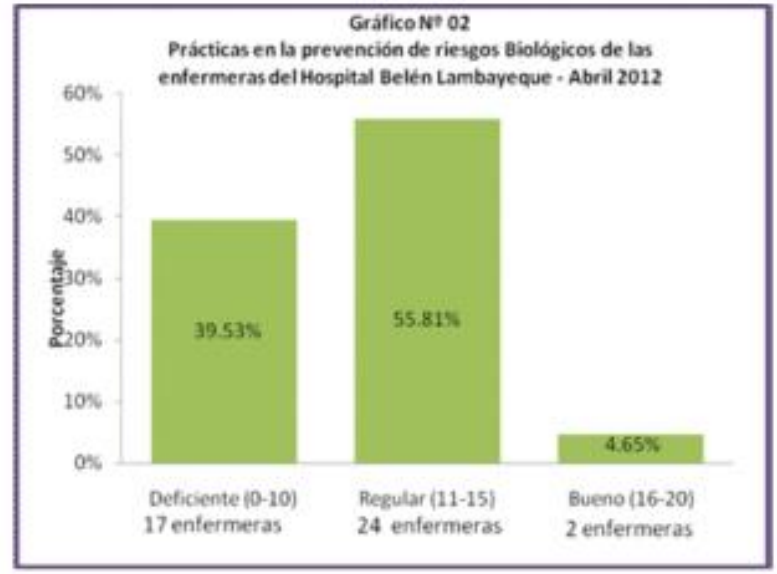

Fuente: Cuestionario realizados a las enfermeras del hospital Provincial Docente BelènAbril 2012.

El resultado del gráfico $\mathrm{N}^{\circ} 02$. Indica que solo el $4.65 \%$ de las enfermeras que laboran en el hospital Belén, tienen una práctica buena en la prevención de riesgos biológicos, y el 39.53\% tienen una práctica deficiente.

\section{Análisis de relación entre Conocimientos y Prácticas.}

Para evaluar la existencia o no de relación entre conocimientos y prácticas en la prevención de riesgos biológicos en las enfermeras, se construyó una tabla y a partir de ella se realizó una prueba de independencia mediante el chi- cuadrado.

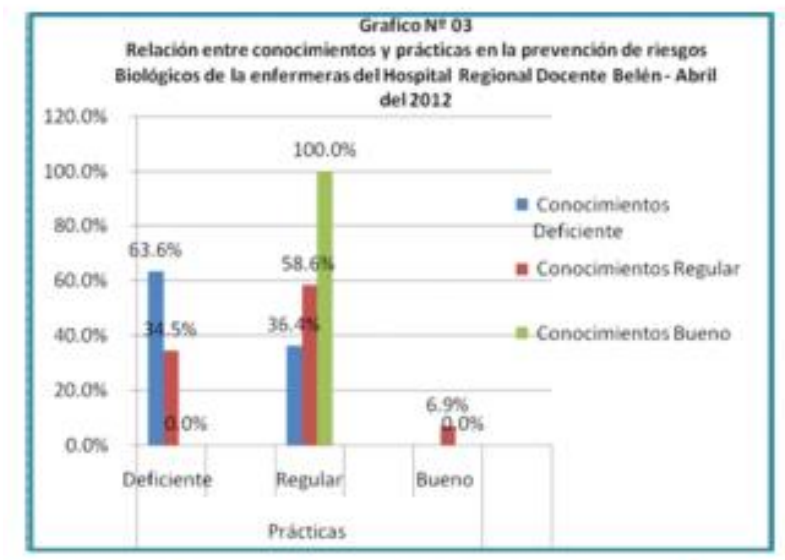

Fuente: Cuestionario realizados a las enfermeras del hospital Provincial Docente BelènAbril 2012.

El valor calculado de Chi cuadrado es 5.79 y el $p$ valor asociado, con 4 grados de libertad es 0.2153 , que resulta ser mayor que el nivel de significancia á $=0.05$, este resultado nos permite observar que no existe una relación significativa entre 
conocimientos y las prácticas de la enfermeras involucradas en la investigación.

Se han calculado también las medidas de asociación entre las variables bajo estudio a través del coeficiente de Cramer, que alcanza el valor de 0.259 , que muestra también una relación muy baja que es coherente con el resultado de la prueba de independencia.

\section{Análisis y discusión}

En el gráfico $\mathrm{N}^{\circ} 01$ : Conocimiento en la prevención de riesgos biológicos de las enfermeras del Hospital Provincial Docente Belén- Abril 2012, se observa que de 43 enfermeras (100\%) que laboran en el hospital Belén-Lambayeque, el $67.44 \%$ tiene un conocimiento regular, $25.58 \%$ un conocimiento deficiente y solo el $6.98 \%$ tienen Conocimiento bueno en la prevención de riesgos biológicos.

Este resultado difiere de la investigación realizada en Perú por Mestanza Vargas en el 2008, denominada "Relación entre conocimientos y prácticas sobre las medidas de bioseguridad en el profesional de enfermería de sala de operaciones del hospital nacional Hipólito Unanue- 2008, donde las medidas de bioseguridad en sala de operaciones se observa que de un total de 25(100\%) enfermeras, 22 (88\%) conocen, mientras que 3 (12\%) no conocen sobre las medidas de bioseguridad. ${ }^{11}$

Al analizar los resultados del estudio realizado en el hospital Belén, se observó que las enfermeras tienen regular conocimiento en la prevención de riesgos biológicos, a diferencia de la investigación realizada por Mestanza Vargas, donde el mayor porcentaje de profesionales en enfermería que laboran en el hospital Hipólito Unanue conoce sobre las medidas de bioseguridad, situación que debería llevar a reflexionar y promover una rápida solución, por parte de las enfermeras, porque ellas conocen estrictamente sobre las medidas de prevención en riesgos biológicos y así evitar el incremento de las infecciones intrahospitalarias que podrían conllevar a enfermedades mortales; se deduce entonces que la implementación de las medidas de prevención es la estrategia primaria necesaria para el control de infecciones nosocomiales en su práctica del cuidado de la salud y la vida. ${ }^{12}$

Cabe mencionar además que la carencia de conocimiento y de información, determina comportamientos equivocados y erróneos. ${ }^{13} \mathrm{Se}$ concluye por tanto que el conocimiento es el elemento más importante que posee una enfermera para poder desarrollar la percepción de riesgo necesaria para proteger la propia salud e incorporarlos a sus prácticas profesionales.

En el gráfico $\mathrm{N}^{\circ}$ 02: Prácticas en la prevención de riesgos biológicos de las enfermeras del hospital Provincial Docente Belén Abril-2012, el 4.65\% de las enfermeras que laboran, tuvieron una práctica buena en la prevención de riesgos biológicos, mientras que el $55.81 \%$ una práctica regular y el 39.53\% una práctica deficiente. Al respecto, Canchán. S y Tapia, Z., con la investigación denominada "Relación entre el nivel de conocimientos y prácticas sobre medidas de bioseguridad de las enfermeras de emergencia del hospital central militar, Lima. 2006". Obtuvieron como resultado que de un total de 22 enfermeras el $81.8 \%$ (18), realizan una práctica deficiente, el $13.6 \%$ (3) regular y sólo el $4.5 \%$ (1) obtuvo el nivel bueno. ${ }^{14}$ Estos resultados evidenciaron que en ambas investigaciones del hospital BelénLambayeque, y el hospital Militar de Lima, las enfermeras no tienen buenas prácticas en la prevención de riesgos biológicos, siendo un resultado preocupante, puesto que las prácticas asistenciales con respecto a los riesgos biológicos son esenciales en el cuidado de enfermería, porque así contribuyen a disminuir las enfermedades infectocontagiosas.

Aquí cabe señalar que las deficientes prácticas en el manejo de riesgos biológicos aumentan los accidentes laborales en el sector sanitario y desde 
el punto de vista preventivo, los accidentes no son nunca eventos fortuitos, existiendo siempre factores (controlables y evitables) que aumentan el riesgo de su aparición.

De ahí la importancia de establecer procedimientos de trabajo adecuados, equipos de seguridad y adoptar medidas de protección colectiva o individual tendientes a evitar o minimizar el riesgo de accidente biológico. ${ }^{15}$ Además la práctica de la enfermería está dirigida al bienestar social en coadyuvancia con otras disciplinas: Su razón de ser y/o objetivo es el cuidado a la persona en las dimensiones: física, mental, emocional, social y espiritual, por lo que requiere aplicar las competencias propias con el fin de prevenir, promover, conservar y recuperar la salud de quien cuida, para lo cual requiere de fundamentos científicos propios, cristalizados en el proceso enfermero que guíe en forma sistematizada, planeada, y organizada su quehacer diario. ${ }^{16}$

De esta manera las buenas prácticas buscan contribuir a mejorar la acción preventiva en los centros de trabajo expuestos a riesgos biológicos, mediante la aplicación de acciones que incidan tanto en la organización de la prevención así como en la planificación de acciones tendentes a disminuir la incidencia de aquellos factores de riesgo más frecuentes en el sector de actividad. ${ }^{17,18}$

El gráfico $\mathrm{N}^{\circ}$ 03: Relación entre conocimientos y prácticas en la prevención de riesgos Biológicos de la enfermeras del hospital Provincial Docente Belén - Abril del 2012, mediante la prueba de chicuadrado, cuyo valor calculado fue 5.79 y el pvalor asociado, con 4 grados de libertad es 0.2153 , resultó ser mayor que el nivel de significancia á $=$ 0.05 , este resultado permitió observar que no existe una relación significativa entre conocimientos y las prácticas de la enfermeras involucradas en la investigación. Así mismo se calcularon también las medidas de asociación entre las variables bajo estudio a través del coeficiente de Cramer, que alcanza el valor de
0.259 , mostrando una relación muy baja que es coherente con el resultado de la prueba de independencia.

Al respecto Cuyubamba, realizó una investigación denominada "Conocimientos y actitudes del personal de salud hacia la aplicación de las medidas de bioseguridad en el Hospital "Feliz Mayorca Soto", Tarma, donde demostró que la relación entre ambas variables no es significativa según el análisis de validación de la prueba Chi cuadrado con una probabilidad de 0.05 y con un grado de libertad 2.19

En este sentido, al analizar las investigaciones anteriormente expuestas se evidencia que no existe relación entre las variables conocimientos y prácticas, reflejándose que el conocer las medidas de prevención, no implica necesariamente su ejecución en las actividades laborales, lo cual significa que para lograr una práctica favorable no solo es necesario el conocimiento sino también es indispensable que los enfermeros tomen conciencia de la función que realizan en la atención de los pacientes para evitar el riesgo de adquirir y/o transmitir enfermedades e infecciones intrahospitalarias. ${ }^{20}$

Está claro que a un mayor nivel de conocimientos los profesionales en enfermería deben tener una correcta aplicación de las medidas para la prevención de riesgos biológicos, no obstante su aplicación no solo dependen de un alto conocimiento de las mismas; sino también de la responsabilidad de los propios enfermeros, porque de nada servirá, poseer conocimientos en cantidad, si no se ofrece un servicio y un trabajo de calidad en el cuidado a los pacientes que acuden al hospital para mejorar su salud.

Al obtener estos resultados de la relación entre ambas variables, es notorio que urge una capacitación para la prevención de riesgos biológicos, con el afán de lograr un mejor cuidado. Toda capacitación implica aplicar y preparar conocimientos preferentemente teóricos y de 
carácter general a fin de incrementar, mejorar y corregir el desempeño profesional, para cumplir funciones con eficacia, eficiencia y seguridad en la organización laboral. 15 Es decir cada enfermera conoce las consecuencias de manipular agentes nocivos para su salud y tiene la alternativa de prevenirlos para evitar que estos se incrementen. ${ }^{21}$

\section{Conclusiones}

La mayoría de profesionales de enfermería en estudio, tienen un conocimiento regular en la prevención de riesgos biológicos; con respecto a este porcentaje es una cifra que debería llevar a reflexionar y promover una rápida solución, se sabe que la carencia de conocimiento, determina comportamientos equivocados y erróneos. siendo preciso incrementar conocimientos para obtener un buen nivel siendo fundamental para proteger la salud e integridad física de sí misma como también del paciente.

En cuanto al cumplimiento de la práctica en la prevención de riesgos biológicos existen profesionales de enfermería que no realizan prácticas adecuadas. El mayor porcentaje de enfermeras realiza una práctica regular representado por el $55.81 \%$ y el $39.53 \%$ tienen una práctica deficiente, siendo necesario mejorarla, monitorizando y evaluando sus actividades. Cabe mencionar que las deficientes prácticas en cuanto al manejo de riesgos biológicos aumentan los accidentes laborales en el sector sanitario.

Al establecer la relación entre conocimiento y la práctica en la prevención de riesgos biológicos de las enfermeras del Hospital Belén- Lambayeque, se aplicó la prueba de chí- cuadrado mediante el cual se demostró que estadísticamente no existe relación significativa, observándose que el conocer sobre la prevención de riesgos biológicos no implica su práctica en las actividades laborales.

Para finalizar, los conocimientos, las buenas prácticas en la prevención de riesgos biológicos y la concientización de los enfermeros ocupacionalmente expuestos son requisitos indispensables para disminuir la accidentalidad por riesgos biológicos en las enfermeras.

\section{Bibliografía}

1. Oficina Internacional del trabajo. Seguridad y salud en el Trabajo. Primera edición. Ginebra; 2009.

2. Ministerio de salud, Dirección General de Salud Ambiental- DIGESA. Manual de salud ocupacional. Lima: 2005.

3. Organización Internacional del Trabajo (OIT). 2011 [Acceso 25 de Agosto 2011] Disponible: htt://www.un.org/org/spanish/new/fullstorynew. asp?NewID=20818.

4. Nuñez Z, Ramírez D. Características epidemiológicas de los accidentes laborales punzocortantes y de exposición mucocutánea en el personal asistencial de enfermería del hospital Alberto Sabogal Sologuren - Es salud. [Revista Recién 2005], [acceso el 20 de setiembre del 2011]. Disponible en http://www.cep.org.pe/cicep/revista/volumen 1/c ap\%2037-42.pdf.

5. Ancco N. Factores que intervienen en la aplicación de medidas de bioseguridad según el profesional de enfermería del Servicio de Sala de Operaciones del Hospital Nacional Dos de Mayo. Tesis para optar el título de enfermería médico Quirúrgica. Lima .2006.

6. Nuñez C, Souza O. El papel de las enfermeras en la prevención de accidentes con material biológico contaminados. 2006. [acceso el 22 de setiembre del 2011]. Disponible en http://botinfo.net/ $p=8068$.

7. Téllez J; Tovar M. Medidas de bioseguridad que aplica el profesional de enfermería y la accidentabilidad laboral en la unidad quirúrgica, 
hospital "Dr. José María Vargas". [Tesis de Bachiller]. Universidad central de venezuela; 2007.

8. Bernal T. Metodología de la investigación. Segunda edición. México: Editorial PearsonEducación; 2006.

9. Hernández R, Fernández C, Baptista P. Metodología de la investigación. Cuarta edición. México: Editorial Mc Graw-Hill Interamericana; 2006.

10. Silva R, Brain L. Validez Y. Confiabilidad Del Estudio Socioeconómico. México: Formación gráfica S.A. de C.V; 2006.

11. Mestanza V. Relación entre conocimientos y prácticas sobre las medidas de bioseguridad en el profesional de enfermería de sala de operaciones del hospital nacional Hipólito Unanue -[Tesis Para optar el Título de Especialista en Enfermería en Centro Quirúrgico]. Universidad San Marcos-Perú; 2008.

12. Ministerio de Salud -MINSA Protocolo. Conocimientos, actitudes y prácticas. Primera edición. Lima $2005 . \quad$ Disponible http:/ / www.minsa.gob.pe/pvigia/publicaciones /infecciones\%20intrahosp.

13. Julca N, García D. Conocimientos de Bioseguridad Hospitalaria en las internas (os) de Enfermería. Huaraz - 2009. [Tesis de Bachiller]. Universidad Católica Los Ángeles de Chimbote. 2009.

14. Canchán S., Tapia Z. Relación entre el nivel de conocimientos y prácticas sobre medidas de bioseguridad de las enfermeras de emergencia del hospital central militar, Lima. 2006. Rev. de Ciencias de la Salud 2:1 2007) Disponible http:/ /posgrado.upeu.edu.pe/revista/filev2/68-72.pdf.

15. Alterio A J, Pérez L H, Medina S C. Nivel de conocimiento y percepción de riesgos biológicos laborales en el personal de enfermería del hospital central universitario "Dr. Antonio maría pineda".
Barquisimeto, Estado Lara. 2.004. [Revista en internet]*2005 Octubre-Diciembre. [Acceso 20 de agosto de 2012]; 7(2). Disponible http: bibmed.ucla.edu.ve/cgiwin/bealex.exe.Boletinmé dico.

16. Murilla M. Competencias de la profesión de enfermería. [Sede Web]*. Cali, Valle del Cauca, Colombia: Epistemología del cuidado; 2010. [acceso 01 de septiembre del 2012]. Disponible http//epistemologíadelcuidado.blogspot.com/20 10/05/competencias de la profesión.

17. Umivale. Mutua de Accidentes de Trabajo y Enfermedades Profesionales. Código de buenas prácticas preventivas exposición al riesgo biológico. N 15).

18. Heluane R, Hacen T. Accidentes por Contacto con Material Biológico. Análisis de sus Determinantes. Departamento investigación/Facultad Medicina, Universidad Nacional de Tucumán / 4000, Tucumán, Argenti. Disponible

http:/ / www.prevencionintegral.com/Articulos / @Datos/_ORP2007/0663.pdf

19. Cuyubamba N. Conocimientos y actitudes del personal de salud, hacia la aplicación de las medidas de bioseguridad del Hospital "Felix Mayorca Soto". Tarma - Perú 2005 [Tesis de Bachiller]. Disponible en: http:/ / ateneo.unmsm.edu.pe/ateneo/handle/ 123 $456789 / 2190$.

20. Navaz SP. La prevención del riesgo de accidente biológico en los profesionales sanitarios. Rev Adm Sanit (España). 2004; 2(4):663-72

21. Salazar M. Conocimientos del personal de enfermería sobre las medidas de bioseguridad en las técnicas de administración de medicamentos. Guatemala [Tesis de Bachiller]. Universidad de san Carlos de Guatemala 2008. 